\title{
DEZ ANOS DE CRIAÇÃO DOS INSTITUTOS FEDERAIS DE EDUCAÇÃO, CIÊNCIA E TECNOLOGIA: SOBRE A OFERTA DE LICENCIATURAS NAS ÁREAS DE CIÊNCIAS E MATEMÁTICA
}

\author{
Ten Years of Creation of Federal Institutes of Education, Science and Technology: \\ about the Offer of Teacher Training Courses in the Areas of Science and Mathematics
}

\author{
Edenmar Guilherme Dall'Agnoll Zacaria* \\ Paulo Vinícius Rebeque** \\ Fernanda Bartoly Gonçalves de Lima***
}

Resumo: Passados dez anos da criação dos Institutos Federais de Educação, Ciência e Tecnologia (IF), apresentamos neste artigo o quantitativo dos cursos existentes nestas instituições, com especial atenção para as licenciaturas em Ciências da Natureza, Ciências Biológicas, Física, Química e Matemática. Tal motivação nos surgiu devido, por um lado, ao contexto de criação dos IF, originalmente instituições de ensino voltadas para a educação técnica e profissional, e, por outro lado, à Lei de Criação dos IF, que exige uma reserva $20 \%$ de suas vagas para cursos de formação de professores, sobretudo nas áreas de Ciências e Matemática. Neste cenário, investigamos o espaço que as licenciaturas ocupam nos IF a partir de consultas a Plataforma Nilo Peçanha (PNP) - ano base de 2018, ambiente virtual de disseminação das estatísticas oficiais dos IF. Com base no quantitativo total de cursos existentes nos IF, identificamos uma maior oferta para os cursos técnicos, cerca de $51 \%$ do total de cursos, e um expressivo crescimento das licenciaturas, cerca de $7 \%$ do total de cursos. Ademais, observamos que há 26 diferentes licenciaturas em funcionamento nos IF, sendo que aproximadamente $62 \%$ desse total são nas áreas de Ciências e Matemática. Com isso, temos que, passada a primeira década, a característica histórica das instituições que deram origem aos IF tem sido preservada, pois há maior oferta para os cursos técnicos e, ao mesmo tempo, a oferta de licenciaturas cresceu e se afirmou na matriz institucional dos IF.

Palavras-chave: Institutos Federais. Licenciaturas. Ciências e Matemática.

Abstract: Ten years after the creation of the Federal Institutes of Education, Science and Technology (FI), we present in this article the number of existing courses in these institutions, with special attention to teacher training courses in Natural Sciences, Biological Sciences, Physics, Chemistry and Mathematics. This motivation has arisen for us, on the one hand, due to the context of creation of FI, originally educational institutions focused on technical and

\footnotetext{
*Licenciado em Física. Professor da Educação Básica do Estado de Santa Catarina. edenmarzacaria@outlook.com.

** Doutor em Ensino de Física. Professor do Instituto Federal de Educação, Ciência e Tecnologia do Rio Grande do Sul - IFRS. paulo.rebeque@bento.ifrs.edu.br

*** Doutora em Educação. Professora do Instituto Federal de Educação, Ciência e Tecnologia de Brasília - IFB. fernanda.lima@ifb.edu.br
} 
professional education, and, on the other hand, to the Law of Creation of the FI, that requires a reservation of $20 \%$ of their vacancies for teacher training courses, especially in the areas of Science and Mathematics. In this scenario, we investigate the space that undergraduate courses occupy in the FI from consultations to the Nilo Peçanha Platform (NPP) - base year 2018, virtual environment for dissemination of official FI statistics. Based on the total number of courses available in the FI, we identified a greater offer for technical courses, about $51 \%$ of the total number of courses, and an expressive growth of undergraduate courses, about $7 \%$ of the total number of courses. Furthermore, we observed that there are 26 different degrees in operation in the FI, which approximately $62 \%$ are in the areas of Science and Mathematics. Therefore, after the first decade, the historical characteristics of the institutions that gave rise to the FI has been preserved, since there is a greater offer for technical courses and, at the same time, the offer of undergraduate courses has grown and established itself as an institutional matrix of the FI.

Keywords: Federal Institutes. Teacher Training Courses. Science and Mathematics.

\section{Introdução}

No dia 29 de Dezembro de 2008, o então Presidente Luiz Inácio Lula da Silva sancionou a Lei $n^{\circ}$ 11.892/2008 (BRASIL, 2008) que instituiu a Rede Federal de Educação Profissional, Científica e Tecnológica (RFEPCT) e criou os Institutos Federais de Educação, Ciência e Tecnologia (IF) ${ }^{1}$. Criadas como instituições de educação básica, profissional e superior, os IF representam uma nova matriz institucional, em tese, revolucionária na educação brasileira. Em especial pelo seu caráter vertical, que permite abrigar no mesmo ambiente educacional diferentes níveis e modalidades de ensino, e por sua estrutura multicampi, que viabilizou um processo intenso de expansão ${ }^{2}$ (JURACY, 2009).

Em essência, os IF possuem como pilar as primeiras escolas de caráter profissional no Brasil, inauguradas em 1909, que, após inúmeros processos de reorganização, tornaram-se em 1994 os Centros Federais de Educação Tecnológica (CEFET) ${ }^{3}$. Conforme dito, somente em 2008, com a Lei ${ }^{\circ}$ 11.892/2008 (BRASIL, 2008), os IF foram criados e inseridos na atual RFEPCT (BRASIL, 2009a). Ou seja, por um lado, os primeiros campi dos IF, em sua maioria, originaram-se de instituições consolidadas na formação profissional e tecnológica e que recém ofertavam cursos de nível superior, os CEFET. Por outro lado, no acelerado processo de expansão, muitos dos novos campi dos IF foram criados em um cenário de déficit de professores e funcionários e notável falta de infraestrutura (FRIGOTTO, 2018).

Quanto aos tipos de cursos, por força de sua própria Lei de criação (BRASIL, 2008), os IF devem garantir a oferta de $50 \%$ de suas vagas para cursos de educação profissional técnica

\footnotetext{
1 A RFEPCT é formada pelos Institutos Federais de Educação, Ciência e Tecnologia (IF), pela Universidade Tecnológica Federal do Paraná (UTFPR), pelos Centros Federais de Educação Tecnológica (CEFET - RJ e CEFET - MG) e pelas Escolas Técnicas vinculadas às Universidades.

${ }^{2}$ Para termos uma ideia quantitativa da expansão de campi dos IF, logo de início, em Dezembro de 2008, foram criados 38 IF que somados agregavam 312 campi em todo o território nacional (JURACY, 2009). Passados dez anos, os mesmos 38 IF somam 593 campi.

3 Em 2009, ano do centenário da RFEPCT, o Ministério da Educação (MEC) inaugurou em seu sítio eletrônico uma página contendo diversas informações históricas e legislativas sobre essa Rede. Destacamos "A Linha do Tempo": http://portal.mec.gov.br/setec/arquivos/centenario/linha.pdf. Acesso em 12 de março de 2020.
} 
de ensino médio, mantendo, assim, a principal característica das instituições que deram origem aos IF: o ensino profissional. Para além de cursos técnicos, os IF também possuem cursos de formação inicial e continuada (FIC) de trabalhadores (denominados cursos de qualificação profissional), de graduação e pós-graduação lato sensu e stricto sensu, afirmando a verticalidade do ensino neste ambiente de acadêmico.

De fato, a variedade de cursos existentes nos IF, em seus diferentes níveis e modalidades de ensino, evidencia o ineditismo destas instituições no sistema educacional. Neste cenário, com a primeira década de existência recém completada, não há exagero em afirmarmos que os IF representam a mais significativa política no campo da educação pública brasileira do século $\mathrm{XXI}$, sendo, tanto em seu conjunto nacional, quanto nas peculiaridades de seus campi, objeto de inúmeras pesquisas (CASTIONI; SOUZA, 2012; FRIGOTTO, 2018; SHIGUNOV NETO; SILVA; FORTUNATO, 2018).

No domínio da graduação, vale destacarmos as licenciaturas, pois os IF têm a responsabilidade de ofertarem $20 \%$ de suas de vagas nestes cursos, privilegiando as áreas de Ciências e Matemática e da educação profissional. Em um primeiro olhar, ao considerarmos a problemática histórica da escassez de professores no Ensino Médio no Brasil (GATTI; BARRETO; ANDRÉ, 2011), com destaque para a época de criação dos IF (BRASIL, 2009b) e nas áreas de Ciências da Natureza e Matemática (ARAUJO; VIANNA, 2011), a obrigação dos IF em oferecer cursos de licenciaturas aparenta surgir para contribuir na "resolução desse problema". Porém, ao recordarmos que os IF possuem um histórico particular relacionado à educação profissional, temos que considerar as implicações dessas instituições enquanto novo locus de formação docente (LIMA, 2016), bem como a expansão das licenciaturas em outros contextos educacionais, em especial o predomínio de licenciaturas a distância oferecidas em instituições privadas (DINIZ-PEREIRA, 2015).

Diante do amplo e complexo contexto dos IF, daremos neste artigo especial atenção a obrigatoriedade dessas instituições na oferta de cursos de licenciatura, sobretudo nas áreas de Ciências e Matemática. Isto porque, mesmo com pouca experiência na formação de professores, os IF surgiram com a responsabilidade de ofertarem cursos de licenciaturas (ARAÚJO; MOURÃO, 2018). Nesse sentido, acreditamos ser importante um levantamento quantitativo dos cursos de licenciaturas nos IF, especialmente para termos uma percepção de como ocorreu a expansão destes cursos na primeira década de existência destas instituições.

Dessa maneira, com o objetivo de identificarmos os cursos existentes no conjunto dos $38 \mathrm{IF}$, empreendemos um olhar inicial para os tipos de cursos e seus respectivos quantitativos de oferta, para, então, destacarmos as licenciaturas nas áreas de Ciências e Matemática, quais sejam: Ciências da Natureza, Ciências Biológicas, Física, Química e Matemática.

Para o desenvolvimento de nossa pesquisa, em primeiro lugar, consultamos os dados disponíveis, referentes ao ano de 2018, na Plataforma Nilo Peçanha (PNP), base de dados oficial da RFEPCT e, naturalmente, dos IF. Com isso, organizamos os números de cursos ofertados em todos os IF, destacando os tipos de cursos e suas principais características. Na sequência, avançando para as licenciaturas, delimitamos os cursos de Ciências da Natureza, Ciências Biológicas, Física, Química e Matemática, segundo o quantitativo destes em cada região do país. Por fim, a partir de um olhar histórico sobre o processo de criação dos IF, assim como do complexo campo da formação de professores, tecemos algumas reflexões sobre a consolidação e os desafios para os cursos de licenciaturas na matriz institucional dos IF. 


\title{
2 Procedimentos Metodológicos: coleta e organização de dados
}

A PNP é o ambiente virtual de disseminação das estatísticas oficiais da RFEPCT ${ }^{4}$ (BRASIL, 2018a). Sua última publicação, 15 de março de 2019, exibe diversas informações acadêmicas e de gestão pública sobre toda a RFEPCT referentes ao ano de 2018. Para além, a PNP é um importante instrumento de produção e análise estatística da RFEPCT, regido por rigoroso processo teórico e metodológico ${ }^{5}$ (MORAES et al., 2018).

Conforme a abrangência da RFEPCT, esclarecemos as nomenclaturas que delinearam nossa coleta de dado centrada nos IF, exibidas no glossário da PNP, nomeadamente:

\begin{abstract}
Cursos: conjunto de atividades educativas formais que constituem um perfil de formação. Além da denominação dos cursos, considera-se uma diferenciação sobre Tipos de cursos, Tipos de Oferta, Modalidade de Ensino e Turno.

Tipo de curso: categorização transversal utilizada para diferenciar os cursos existentes em seus diferentes níveis e graus. No caso dos IF, estão previstos os seguintes cursos: Ensino Médio (Básico Propedêutico), Formação Inicial e Continuada (FIC) - também denominados de cursos de Qualificação Profissional, Técnico, Tecnologia, Licenciatura, Bacharelado, Especialização (lato Sensu), Mestrado Profissional, Mestrado, Doutorado e Doutorado Profissional.

Tipo de Oferta: categorização transversal utilizada para diferentes formas de oferta dos cursos Técnicos e FIC. As seguintes categorias são previstas: integrado, PROEJA integrado, concomitante, PROEJA concomitante, e subsequente ${ }^{6}$.

Modalidade de Ensino: modo de desenvolvimento das atividades acadêmicas do curso, podendo ser presencial ou a distância (EaD).

Turno: período em que o estudante cursa a maior parte das aulas, podendo ser matutino, vespertino, noturno ou integral. Essa diferenciação não se aplica aos cursos na modalidade EaD. ${ }^{7}$
\end{abstract}

Neste artigo, daremos atenção aos dados referentes ao número de cursos em funcionamento no ano de 2018 nos IF, especialmente para os cursos presenciais de licenciatura nas áreas de Ciências e Matemática. Para tal, consultamos na PNP, item 1.1 Instituições, o quantitativo total de cursos existentes no conjunto dos 38 IF (Organização Acadêmica). Na sequência, item 1.2 Tipo de Curso, chegamos aos números de cursos em funcionamento, de vagas, de ingressantes e de concluintes, para cada tipo de curso. Prosseguindo, miramos no

\footnotetext{
${ }^{4} \mathrm{O}$ endereço eletrônico da PNP é https://www.plataformanilopecanha.org/. Acesso em 20 de março de 2020.

${ }^{5}$ Mesmo não sendo nosso objetivo uma análise sobre o percentual de vagas ofertadas nos IF para os tipos de cursos em funcionamento, importa-nos ressaltar o marco regulatório da PNP: Portaria SETEC $\mathrm{n}^{\circ} 51 / 2018$, que define conceitos e estabelece regras para o cálculo de indicadores na PNP (BRASIL, 2018b) e Portaria $\mathrm{n}^{\circ} 1.162 / 2018$, que regulamenta o conceito de Aluno-Equivalente e de Relação Aluno por Professor (BRASIL, 2018c).
}

\begin{abstract}
${ }^{6}$ Em resumo, nos cursos Técnicos integrados o estudante realiza o Ensino Médio regular e o curso Técnico como sendo um único curso, possuindo, assim, uma única matrícula; nos cursos Técnicos concomitantes o estudante possui duas matrículas, uma para o curso Técnico e outra para o Ensino Médio regular, podendo ser o Ensino Médio cursado em outra instituição; nos cursos Técnicos subsequentes o estudante precisa, necessariamente, ter concluído o Ensino Médio. Por fim, inseridos no Programa Nacional de Integração da Educação Profissional com a Educação Básica na Modalidade de Educação de Jovens e Adultos (PROEJA), os cursos PROEJA integrado e PROEJA concomitante abrem possibilidades para Jovens e Adultos concluírem não apenas o ensino médio, mas também aprendem um profissão nos cursos Técnicos.
\end{abstract}

${ }^{7}$ Disponível em <http://plataformanilopecanha.mec.gov.br/2019.html>. Acesso em 02 de maio de 2020. 
quantitativo de cursos de licenciaturas em geral, para, finalmente, selecionarmos as licenciaturas em Ciências da Natureza, Ciências Biológicas, Física, Química e Matemática.

Destacamos a facilidade de coleta de dados na PNP, uma vez que essa ferramenta online oferece boa interatividade e permite aos usuários uma rápida consulta sobre diversas informações da RFEPCT. Apenas para fins de ilustração, apresentamos na Figura 1 uma imagem da PNP, tendo selecionado o item 1.1 Instituições e feito as seguintes especificações: Instituto Federal como organização acadêmica, licenciaturas para os tipos de cursos e Ciências da Natureza, Ciências Biológicas, Física, Química e Matemática como nome dos cursos.

Figura 1 - Imagem de uma consulta realizada na PNP.

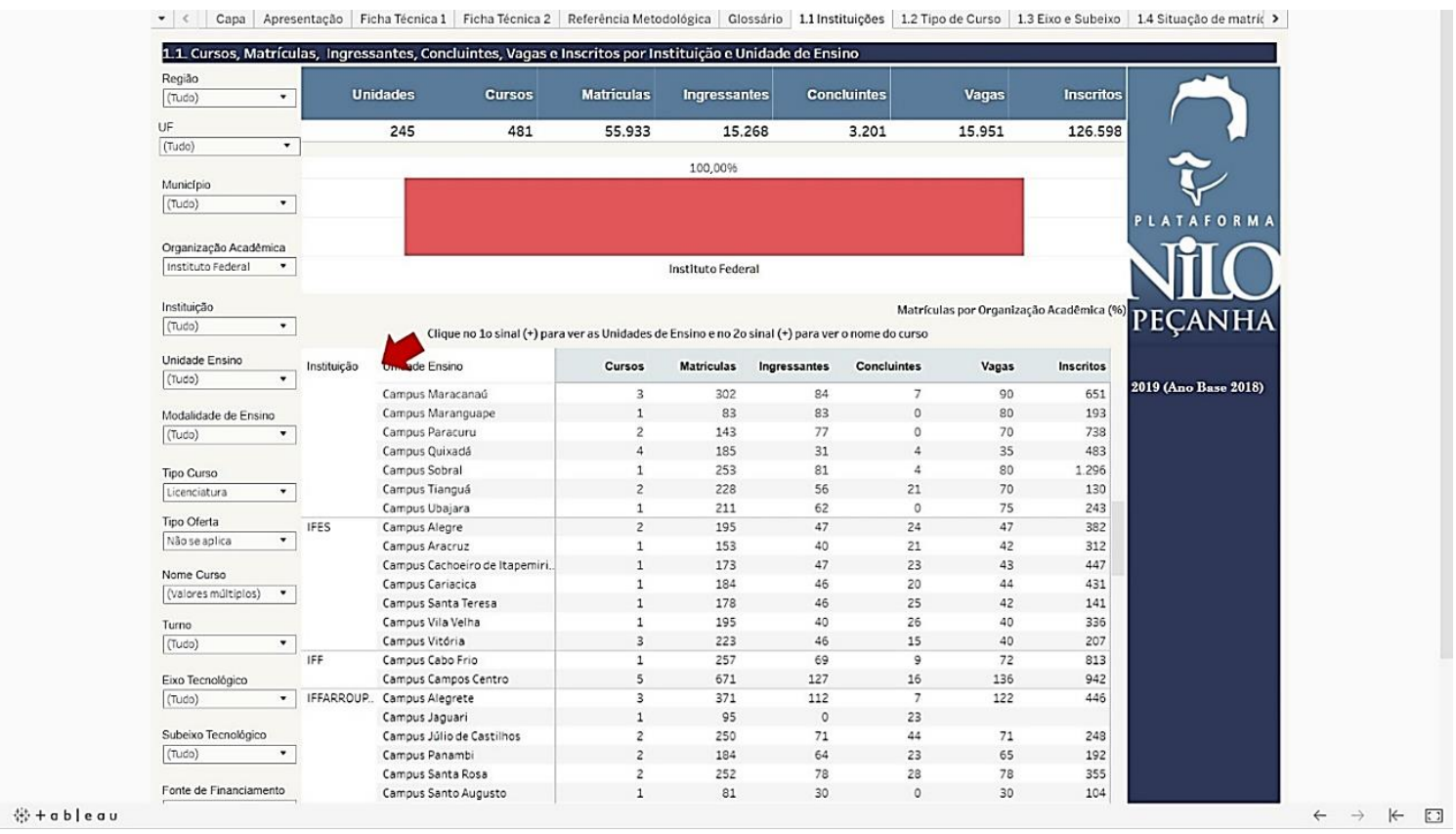

Fonte: PNP - acesso em março de 2020.

\section{Sobre a oferta de cursos nos Institutos Federais de Educação, Ciência e Tecnologia}

O art. $7^{\circ}$ da Lei ${ }^{\circ} 11.892 / 2008$ (BRASIL, 2008) evidencia os objetivos dos IF, dos quais destacamos os referentes aos tipos de cursos:

I - ministrar educação profissional técnica de nível médio, prioritariamente na forma de cursos integrados, para os concluintes do ensino fundamental e para o público da educação de jovens e adultos;

II - ministrar cursos de formação inicial e continuada de trabalhadores, objetivando a capacitação, o aperfeiçoamento, a especialização e a atualização de profissionais, em todos os níveis de escolaridade, nas áreas da educação profissional e tecnológica; [...] VI - ministrar em nível de educação superior:

a) cursos superiores de tecnologia visando à formação de profissionais para os diferentes setores da economia;

b) cursos de licenciatura, bem como programas especiais de formação pedagógica, com vistas na formação de professores para a educação básica, sobretudo nas áreas de ciências e matemática, e para a educação profissional;

c) cursos de bacharelado e engenharia, visando à formação de profissionais para os diferentes setores da economia e áreas do conhecimento; 
d) cursos de pós-graduação lato sensu de aperfeiçoamento e especialização, visando à formação de especialistas nas diferentes áreas do conhecimento;

e) cursos de pós-graduação stricto sensu de mestrado e doutorado, que contribuam para promover o estabelecimento de bases sólidas em educação, ciência e tecnologia, com vistas no processo de geração e inovação tecnológica.

Esses objetivos afirmam a verticalidade do ensino como importante característica dos IF, o que viabilizou, de acordo com a PNP no ano base de 2018, o funcionamento de cursos de nível Médio, Técnico, Qualificação Profissional, Graduação e Pós-Graduação.

Passados dez anos da criação dos IF, realizamos um primeiro levantamento na PNP do quantitativo de campi e do quantitativo de cursos em funcionamento em cada uma de suas 38 instituições. Na Tabela 1, apresentamos em detalhes esses números de acordo com a região do país em que as instituições se situam.

Tabela 1 - Instituições de educação que integram os IF, bem como número de campi e de cursos em funcionamento em cada instituição no ano de 2018.

\begin{tabular}{|c|c|c|c|}
\hline & Nome das instituições de educação que integram os IF & $\begin{array}{l}\mathrm{n}^{\mathbf{0}} \mathrm{de} \\
\text { campi }\end{array}$ & $\begin{array}{c}\mathrm{n}^{0} \mathrm{de} \\
\text { cursos }\end{array}$ \\
\hline \multirow{5}{*}{$\begin{array}{l}\mathbf{C} \\
\mathrm{e} \\
\mathbf{n} \\
\mathbf{t} \\
\mathbf{r} \\
\mathbf{o} \\
- \\
\mathrm{O} \\
\mathrm{e} \\
\mathrm{s} \\
\mathbf{t} \\
\mathrm{e}\end{array}$} & Instituto Federal de Brasília (IFB) & 10 & 216 \\
\hline & Instituto Federal de Goiás (IFG) & 14 & 242 \\
\hline & Instituto Federal Goiano (IFGOIANO) & 12 & 253 \\
\hline & Instituto Federal de Mato Grosso (IFMT) & 19 & 301 \\
\hline & Instituto Federal de Mato Grosso do Sul (IFMS) & 10 & 207 \\
\hline \multirow{11}{*}{$\begin{array}{l}\mathbf{N} \\
\mathbf{o} \\
\mathbf{r} \\
\mathbf{d} \\
\mathrm{e} \\
\mathrm{s} \\
\mathrm{t} \\
\mathrm{e}\end{array}$} & Instituto Federal de Alagoas (IFAL) & 16 & 244 \\
\hline & Instituto Federal da Bahia (IFBA) & 21 & 251 \\
\hline & Instituto Federal Baiano (IFBAIANO) & 15 & 220 \\
\hline & Instituto Federal do Ceará (IFCE) & 32 & 686 \\
\hline & Instituto Federal do Maranhão (IFMA) & 30 & 511 \\
\hline & Instituto Federal da Paraíba (IFPB) & 21 & 242 \\
\hline & Instituto Federal de Pernambuco (IFPE) & 16 & 283 \\
\hline & Instituto Federal do Piauí (IFPI) & 20 & 353 \\
\hline & Instituto Federal do Rio Grande do Norte (IFRN) & 20 & 533 \\
\hline & Instituto Federal de Sergipe (IFS) & 9 & 103 \\
\hline & Instituto Federal do Sertão Pernambucano (IFSERTÃO-PE) & 7 & 201 \\
\hline \multirow{7}{*}{$\begin{array}{l}\mathbf{N} \\
\mathbf{o} \\
\mathbf{r} \\
\mathbf{t} \\
\mathbf{e}\end{array}$} & Instituto Federal do Acre (IFAC) & 6 & 79 \\
\hline & Instituto Federal do Amazonas (IFAM) & 15 & 258 \\
\hline & Instituto Federal do Amapá (IFAP) & 6 & 140 \\
\hline & Instituto Federal do Pará (IFPA) & 18 & 497 \\
\hline & Instituto Federal de Rondônia (IFRO) & 9 & 175 \\
\hline & Instituto Federal de Roraima (IFRR) & 5 & 92 \\
\hline & Instituto Federal do Tocantins (IFTO) & 11 & 147 \\
\hline \multirow{6}{*}{$\begin{array}{l}\mathbf{S} \\
\mathbf{u} \\
\mathbf{d} \\
\mathbf{e} \\
\mathbf{s} \\
\mathbf{t} \\
\mathbf{e}\end{array}$} & Instituto Federal do Espírito Santo (IFES) & 22 & 330 \\
\hline & Instituto Federal Fluminense (IFF) & 12 & 191 \\
\hline & Instituto Federal de Minas Gerais (IFMG) & 18 & 202 \\
\hline & Instituto Federal do Norte de Minas Gerais (IFNMG) & 13 & 423 \\
\hline & Instituto Federal do Rio de Janeiro (IFRJ) & 15 & 151 \\
\hline & Instituto Federal de São Paulo (IFSP) & 37 & 854 \\
\hline
\end{tabular}




\begin{tabular}{c|l|c|c}
\hline & Instituto Federal do Sudeste de Minas (IFSUDESTE-MG) & 10 & 211 \\
\cline { 2 - 4 } & Instituto Federal do Sul de Minas (IFSULDEMINAS) & 9 & 263 \\
\cline { 2 - 4 } & Instituto Federal do Triângulo Mineiro (IFTM) & 9 & 183 \\
\hline $\mathbf{S}$ & Instituto Federal Catarinense (IFC) & 15 & 214 \\
\cline { 2 - 4 } $\mathbf{u}$ & Instituto Federal Farroupilha (IFFARROUPILHA) & 11 & 171 \\
\cline { 2 - 4 } & Instituto Federal do Paraná (IFPR) & 25 & 349 \\
\cline { 2 - 4 } & Instituto Federal do Rio Grande do Sul (IFRS) & 17 & 307 \\
\cline { 2 - 4 } & Instituto Federal de Santa Catarina (IFSC) & 23 & 721 \\
\cline { 2 - 4 } & Instituto Federal Sul-rio-grandense (IFSUL) & 15 & 251 \\
\hline
\end{tabular}

Fonte: dados da PNP organizados pelos autores - ano base de 2018.

Considerando os tipos de cursos existentes nos IF, independentemente do tipo de oferta e da modalidade de ensino, em números absolutos, registramos 4 cursos de Ensino Médio, 5.608 cursos Técnicos, 2.797 cursos FIC, 2.018 cursos de Graduação e 628 cursos de Pós-Graduação. Totalizando, assim, os 11.055 cursos em funcionamento em 2018 nos 593 campi que integram os 38 IF. Em termos de região, temos a Nordeste com a maior presença de campi e de cursos em funcionamento (207 e 3.627), seguida, respectivamente, das regiões Sudeste (145 e 2.808), Sul (106 e 2.013), Norte (70 e 1.388) e Centro-Oeste (65 e 1.219).

Percebemos nestes números que a maior oferta está nos cursos Técnicos, missão prioritária dos IF, dado que sua Lei de criação (BRASIL, 2008) indica a oferta mínima de 50\% de suas vagas para a educação profissional técnica de nível médio. Ainda, os cursos Técnicos conseguem atender diferentes públicos, conforme tipos de oferta. Já para os cursos de Graduação, identificamos três tipos: Bacharelado, Licenciatura e Tecnologia. Destes cursos, as Licenciaturas apresentam maior oferta, o que pode ser uma consequência direta da Lei $\mathrm{n}^{\mathbf{o}}$ 11.892/2008 (BRASIL, 2008) que prevê o mínimo de 20\% das vagas dos IF para cursos de licenciatura, sobretudo nas áreas de Ciências e Matemática. Por fim, a Pós-Graduação possui o menor quantitativo de oferta dentro dos tipos de curso dos IF.

Na Tabela 2, para cada tipo de curso, exibimos os números da PNP para os cursos em funcionamento, as vagas ofertadas, os ingressantes e os concluintes ${ }^{8}$, o ano de referência 2018.

\footnotetext{
${ }^{8}$ Entendemos como vagas ofertadas o número total de vagas disponibilizadas para a fase inicial dos cursos, como ingressante os estudantes que de fato iniciaram um curso e como concluintes o somatório de estudantes que finalizaram as exigências de um curso e que foram diplomados. Ademais, importa-nos esclarecer que há cursos em funcionamento que deixaram de ofertar vagas em 2018 (ou em anos anteriores). Ou seja, mesmo sem ofertar vagas, estes cursos permanecerão em funcionamento até que não haja mais estudantes matriculados.
} 
Tabela 2 - Quantitativo de cursos em funcionamento em 2018, de vagas ofertadas, de ingressantes e de concluintes, no conjunto dos $38 \mathrm{IF}$, de acordo com o tipo de curso.

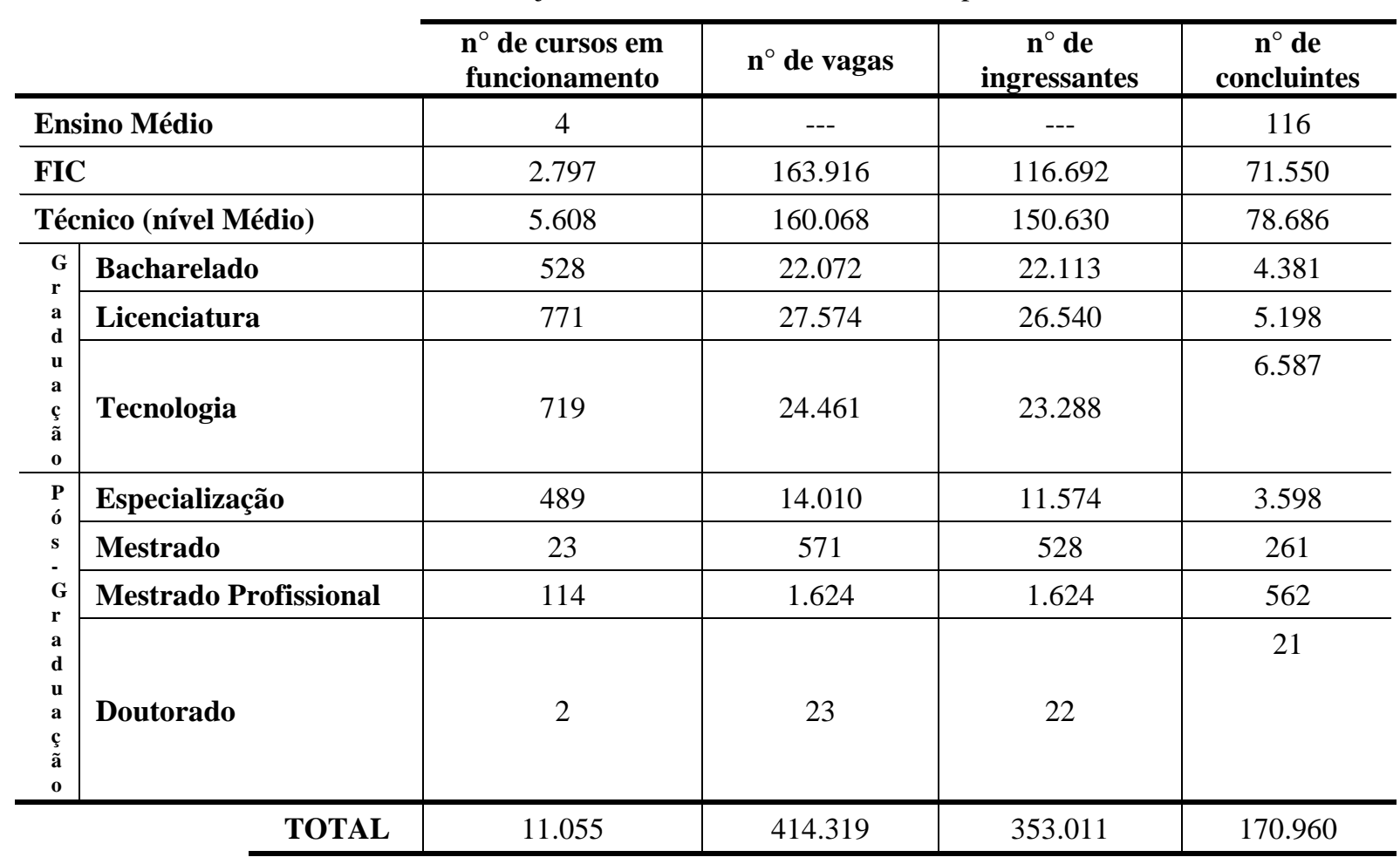

Fonte: dados da PNP organizados pelos autores - ano base de 2018.

O total de cursos em funcionamento no ano de 2018 nos mostra o importante papel dos IF na educação pública brasileira. Em especial, conforme tipos de cursos, de oferta e modalidades de ensino, os IF conseguem atender um público diverso em todo o país, dado que muitos de seus campi estão localizados em cidades/regiões do interior brasileiro.

Em termos do número de cursos existentes nos IF, constatamos a grande influência da Lei $\mathrm{n}^{\mathrm{o}}$ 11.892/2008 (BRASIL, 2008), sendo dos cursos Técnicos a maior oferta, cerca de 51\% do total de cursos. Vale ressaltarmos que os percentuais que acima mencionamos é sobre o quantitativo de cursos em funcionamento, mas que adota-se como parâmetro para o cálculo da oferta de vagas o conceito de aluno-equivalente (BRASIL, 2018c).

Ainda sobre o quantitativo de cursos em funcionamento nos IF, percebemos que os cursos FIC apresentam menor número em relação aos cursos técnico, porém disponibilizam mais vagas. Isso é perfeitamente compreensível quando olhamos para as características desses cursos: voltados para a qualificação da vida profissional, para o mercado de trabalho, com carga horária mínima de 8 horas e máxima de 360 horas. Por fim, as licenciaturas se destacam com a maior oferta entre os cursos de graduação, enquanto na pós-graduação observamos uma tendência para cursos de especialização (lato sensu) e de mestrado profissional (stricto sensu).

A partir desse amplo levantamento sobre os cursos em funcionamento nos IF, faremos na sequência uma análise sobre os cursos de graduação, para, finalmente, mirarmos nas licenciaturas das áreas de Ciências e Matemática. 


\section{Sobre da oferta das Licenciatura em Ciências da Natureza, Ciências Biológicas, Física, Química e Matemática nos IF}

Na Tabela 3 dispomos os quantitativos de cursos de graduação em funcionamento nos IF para cada região do Brasil no ano base de 2018.

Tabela 3 - Quantitativo de cursos de graduação em funcionamento em 2018 no conjunto dos 38 IF, de acordo com o tipo de curso em cada região do país.

\begin{tabular}{l|c|c|c|c}
\cline { 2 - 5 } & $\begin{array}{c}\mathbf{n}^{\circ} \text { de cursos de } \\
\text { bacharelado }\end{array}$ & $\begin{array}{c}\mathbf{n}^{\circ} \text { de cursos de } \\
\text { licenciatura }\end{array}$ & $\begin{array}{c}\mathbf{n}^{\circ} \text { de cursos de } \\
\text { tecnologia }\end{array}$ & $\begin{array}{c}\mathbf{n}^{\circ} \text { de cursos de } \\
\text { graduação }\end{array}$ \\
\hline Centro-Oeste & 79 & 77 & 90 & 246 \\
\hline Nordeste & 126 & 303 & 225 & 654 \\
\hline Norte & 37 & 115 & 103 & 255 \\
\hline Sudeste & 193 & 162 & 160 & 515 \\
\hline Sul & 93 & 114 & 141 & 348 \\
\hline \multicolumn{1}{c|}{ TOTAL } & 528 & 771 & 719 & 2.018 \\
\hline
\end{tabular}

Fonte: dados da PNP organizados pelos autores - ano base de 2018.

Em uma análise englobando todos os cursos existentes nos IF, constatamos que dez anos após sua criação, os cursos de graduação representam 18,25\% do total. Levando em conta o processo histórico de criação dos IF, este percentual nos revela um elevado crescimento desse tipo de curso, com destaque para as licenciaturas: aproximadamente 7\% do total de cursos ou, ainda, $38 \%$ do total dos cursos de graduação.

Ao iniciarmos nossa análise sobre as licenciaturas em funcionamento nos IF, identificamos 26 denominações para estes cursos, quais sejam: Artes Cênicas, Artes Visuais, Ciências Agrárias, Ciências Biológicas, Ciências da Natureza, Ciências Sociais, Computação, Dança, Educação do Campo, Educação Física, Física, Geografia, História, Informática, Intercultural Indígena, Letras (Geral, Língua Estrangeira e Língua Portuguesa), Libras, Educação Profissional, Científica e Tecnológica, Matemática, Música, Pedagogia, Formação Pedagógica Docente, Química e Teatro.

Fazendo uma análise puramente quantitativa sobre as licenciaturas existentes nos IF, percebemos que os cursos de Matemática, Química, Ciências Biológicas e Física são, respectivamente, os cursos com maior quantitativo: 132, 119, 112 e 98 . Ou seja, as licenciaturas em maior número nos IF são das áreas de Ciências e Matemática, revelando alinhamento com a própria Lei de Criação dos IF (BRASIL, 2008). Ademais, podemos contabilizar os 20 cursos de licenciatura em Ciências da Natureza, uma vez que estes cursos, além de formarem professores de Ciências para o Ensino Fundamental, podem habilitar professores para atuarem nas disciplinas de Biologia, Física ou Química no Ensino Médio, conforme projeto pedagógico.

Em percentuais, conforme dissemos, as licenciaturas representam apenas $7 \%$ do total de cursos existentes nos IF. Entretanto, dentre as 26 denominações de licenciaturas, somente as das áreas de Ciências e Matemática representam 62,4\%. Com isso, constatamos que no conjunto dos 38 IF as licenciaturas em geral representam um pequeno percentual, sendo prioridade dada às licenciaturas em Ciências da Natureza, Ciências Biológicas, Física, Química e Matemática.

Pensando na modalidade de ensino das licenciaturas existentes nos IF em 2018, temos que aproximadamente $84 \%$ são cursos presenciais. Este percentual, em valores aproximados, aumenta para as licenciaturas em Ciências da Natureza (100\%), Ciências Biológicas (88\%), Física (96\%), Química (95\%) e Matemática (92\%). Ou seja, há um predomínio na oferta de 
licenciaturas presenciais nos IF, situação oposta ao processo de expansão de licenciaturas em instituições privadas (DINIZ-PEREIRA, 2015). Além disso, dos 648 cursos de licenciaturas presenciais, 358 são ofertados no período noturno, o que corresponde a pouco mais de $55 \%$. Tal percentual é maior para as licenciaturas em Ciências Biológicas (57\%), Física (62\%), Química (62\%) e Matemática (58\%) e menor para Ciências da Natureza (40\%).

Por fim, em números absolutos, temos que das 771 licenciaturas existentes nos IF, 481 são da área de Ciências e Matemática. Para melhor ilustrar a distribuição dos cursos existentes nos IF em cada região do Brasil, apresentamos na Figura 2 (a) o quantitativo de cursos de graduação, licenciaturas em geral (todas as 26 denominações) e licenciaturas em Ciências e Matemática e na Figura 2 (b) um detalhamento para os cursos de Ciências da Natureza, Ciências Biológicas, Física, Química e Matemática.

Figura 2 - Quantitativo de específicos cursos existentes nos IF em 2018 conforme região do Brasil. (a) cursos de graduação, de licenciatura em geral e licenciaturas nas áreas de Ciências e Matemática e (b) cursos de licenciatura em Ciências da Natureza, Ciências Biológicas, Física, Química e Matemática.

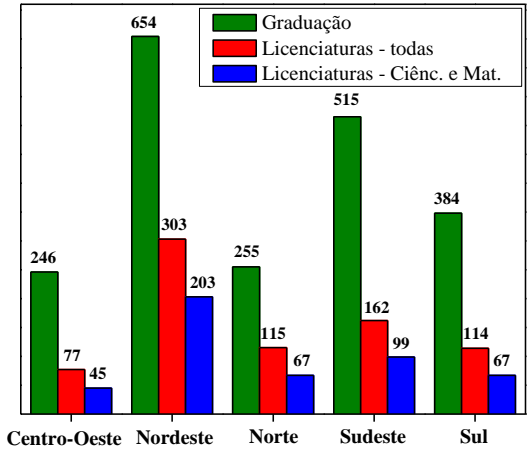

(a)

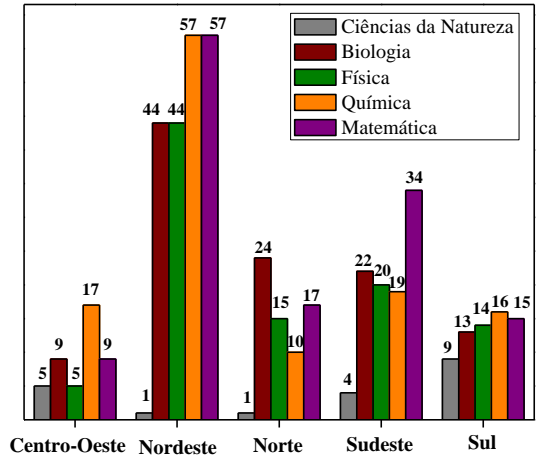

(b)

Fonte: dados da PNP organizados pelos autores - ano base de 2018.

A análise acima empreendida nos revela não apenas o predomínio da região Nordeste, em relação às demais regiões do país, no número de campi e de cursos existentes, mas também no quantitativo de licenciaturas em geral e das áreas de Ciências e Matemática (esta região só não possui um maior quantitativo para o curso de Ciências da Natureza). Isso nos revela que não há uma distribuição proporcional das licenciaturas nas regiões do país, mesmo porque cada um dos 38 IF possuem autonomia na decisão de oferta de cursos. Para aprofundar essa análise ao nível dos estados brasileiros, apresentamos na Tabela 4 os quantitativos de todos os cursos, de todas licenciaturas e das licenciaturas nas áreas de Ciências e Matemática, em funcionamento nos IF no ano de 2018. 
Revista de Educação, Ciência e Tecnologia

Tabela 4 - Números da PNP de todos os cursos existentes nos IF em 2018, das licenciaturas (com o percentual aproximado em relação ao total de cursos) e das licenciaturas em Ciências e Matemática (com o percentual aproximado em relação ao total de licenciaturas), conforme estado da federação.

\begin{tabular}{|c|c|c|c|c|c|c|}
\hline & Estado da Federação & $\begin{array}{c}\mathrm{n}^{0} \text { total de } \\
\text { cursos }\end{array}$ & $\begin{array}{c}\mathrm{n}^{\circ} \text { total de } \\
\text { licenciaturas }\end{array}$ & $(\%)$ & $\begin{array}{l}\mathbf{n}^{\circ} \text { de lic. nas áreas de } \\
\text { Ciências e Matemática }\end{array}$ & $(\%)$ \\
\hline \multirow{5}{*}{$\begin{array}{l}\text { C } \\
\text { e } \\
\text { n } \\
\text { t } \\
\text { r } \\
\text { o } \\
- \\
\text { O } \\
\text { e } \\
\text { s } \\
\text { t } \\
\text { e }\end{array}$} & Distrito Federal & 216 & 16 & 7,4 & 7 & 43,8 \\
\hline & Goiás & 495 & 41 & 8,3 & 20 & 48,8 \\
\hline & Mato Grosso & 301 & 18 & 6,0 & 17 & 94,4 \\
\hline & Mato Grosso do Sul & 207 & 2 & 1,0 & 1 & 50,0 \\
\hline & Total da região & 1.219 & 77 & --- & 45 & --- \\
\hline \multirow{10}{*}{$\begin{array}{l}\mathbf{N} \\
\mathbf{r} \\
\mathbf{r} \\
\mathrm{d} \\
\mathrm{e} \\
\mathrm{s} \\
\mathrm{t} \\
\mathrm{e}\end{array}$} & Alagoas & 244 & 26 & 10,7 & 14 & 53,8 \\
\hline & Bahia & 471 & 33 & 7,0 & 15 & 45,5 \\
\hline & Ceará & 686 & 76 & 11,1 & 48 & 63,2 \\
\hline & Maranhão & 511 & 51 & 10,0 & 41 & 80,4 \\
\hline & Paraíba & 242 & 16 & 6,6 & 6 & 37,5 \\
\hline & Pernambuco & 484 & 28 & 5,8 & 20 & 71,4 \\
\hline & Piauí & 353 & 36 & 10,2 & 34 & 94,4 \\
\hline & Rio Grande do Norte & 533 & 34 & 6,4 & 22 & 64,7 \\
\hline & Sergipe & 103 & 3 & 2,9 & 3 & 100,0 \\
\hline & Total da região & 3.627 & 303 & --- & 203 & --- \\
\hline \multirow{8}{*}{$\begin{array}{l}\mathbf{N} \\
\mathbf{o} \\
\mathbf{r} \\
\mathbf{t} \\
\mathbf{e}\end{array}$} & Acre & 79 & 7 & 8,9 & 7 & 100,0 \\
\hline & Amapá & 140 & 12 & 8,6 & 8 & 66,7 \\
\hline & Amazonas & 258 & 13 & 5,0 & 9 & 69,2 \\
\hline & Pará & 497 & 47 & 9,5 & 24 & 51,1 \\
\hline & Rondônia & 175 & 8 & 4,6 & 6 & 75,0 \\
\hline & Roraima & 92 & 15 & 16,3 & 8 & 53,3 \\
\hline & Tocantins & 147 & 13 & 8,8 & 5 & 38,5 \\
\hline & Total da região & 1.388 & 115 & --- & 67 & -- \\
\hline \multirow{5}{*}{$\begin{array}{l}\text { S } \\
\mathbf{u} \\
\text { d } \\
\text { e } \\
\text { S } \\
\text { t } \\
\text { e }\end{array}$} & Espírito Santo & 330 & 21 & 6,4 & 10 & 47,6 \\
\hline & Minas Gerais & 1282 & 63 & 4,9 & 37 & 58,7 \\
\hline & Rio de Janeiro & 342 & 23 & 6,7 & 14 & 60,9 \\
\hline & São Paulo & 854 & 55 & 6,4 & 38 & 69,1 \\
\hline & Total da região & 2.808 & 162 & --- & 99 & --- \\
\hline \multirow{4}{*}{$\begin{array}{l}\mathbf{S} \\
\mathbf{u} \\
\mathbf{l}\end{array}$} & Paraná & 349 & 21 & 6,0 & 16 & 76,2 \\
\hline & Santa Catarina & 935 & 29 & 3,1 & 19 & 65,5 \\
\hline & Rio Grande do Sul & 729 & 64 & 8,8 & 32 & 50,0 \\
\hline & Total da região & 2.013 & 114 & --- & 67 & --- \\
\hline \multicolumn{2}{|c|}{ TOTAL GERAL } & $\mathbf{1 1 . 0 5 5}$ & 771 & $-\ldots$ & 481 & --- \\
\hline
\end{tabular}

Fonte: dados da PNP organizados pelos autores - ano base de 2018.

Lembramos que por exigência legal os IF devem reservar $20 \%$ de suas de vagas para as licenciaturas (BRASIL, 2008), sendo que o parâmetro adotado para o cálculo da oferta de vagas é o conceito de aluno-equivalente (BRASIL, 2018c). Porém, organizamos na Tabela 4 os números de cursos em funcionamento dos IF em cada estado da federação, segundo os dados da PNP para o ano de 2018. Por isso, também lembramos que neste artigo nos concentramos no levantamento quantitativo das licenciaturas, visto que nossa intenção é buscar compreensão 


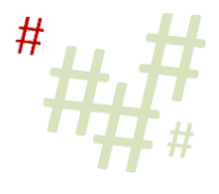

sobre o espaço que estes cursos ocupam dez anos após a criação dos IF, uma instituição historicamente voltada para o ensino técnico e profissional.

Isto posto, percebemos que apenas cinco estados possuem um percentual maior ou igual a $10 \%$ de licenciaturas em relação ao número total de cursos em funcionamento (Roraima, Ceará, Alagoas, Piauí e Maranhão), dos quais quatro pertencem à região Nordeste. Ou seja, os baixos percentuais das licenciaturas em relação ao total de cursos existentes nos IF dão um indicativo de que ainda não foi alcançado a meta de ofertar $20 \%$ das vagas para as licenciaturas. Em contrapartida, em muitos estados identificamos grande alinhamento com a preferência em ofertar licenciaturas nas áreas de Ciências e Matemática (sete estados possuem percentual maior ou igual a 75\%: Acre, Sergipe, Mato Grosso, Piauí, Maranhão, Paraná e Rondônia).

Estes índices nos revelam, por um lado, um baixo quantitativo de cursos de licenciaturas em relação ao total de cursos em funcionamento nos IF e, por outro lado, no âmbito das licenciaturas, grande predomínio dos cursos nas áreas de Ciências e Matemática. Em verdade, não temos conhecimento se há alguma espécie de verificação quantitativa, por parte da RFEPCT, sobre os tipos cursos existentes nos IF com base no conceito de aluno-equivalente. Isto é, embora a Lei $\mathrm{n}^{\circ}$ 11.892/2008 (BRASIL, 2008) estabeleça percentuais para a reserva de vagas (50\% educação profissional técnica e $20 \%$ licenciaturas), passados dez anos de criação dos IF, não nos é perceptível nenhuma forma de controle, ou estabelecimento de prazos, para que estas metas sejam alcançadas.

\section{Considerações Finais: conquistas e desafios para as licenciaturas dos IF}

Conforme temos dito, os IF foram criados e inseridos na RFEPCT após inúmeros processos de reorganização de instituições de ensino consolidadas na formação profissional e tecnológica, o que potencializou uma expansão de oferta do ensino público em todo o país (JURACY, 2009). Sobretudo, os IF representam uma nova matriz institucional na educação brasileira que viabilizou a criação de novos campi para a oferta de uma variedade de cursos (em diferentes níveis e modalidades) em todo o Brasil. Justamente por essas circunstâncias, de possuírem histórico relacionado à educação profissional, uma missão prioritária na formação técnica e tecnológica, Lima (2016) entende os IF como um novo locus de formação docente.

Embora seja assegurada pela Lei $n^{\circ} 11.892 / 2008$ (BRASIL, 2008), a oferta de licenciaturas nos IF não ocorreu neste instante de modo abrupto, pois os CEFET, instituições que deram origem aos IF, já estavam em um processo de inserção de licenciaturas em sua estrutura educacional (LIMA; CURADO SILVA, 2013; ARAÚJO; MOURÃO, 2018). De fato, no ano de 2000 os CEFET passaram a ter autonomia para criarem cursos de formação de professores para as disciplinas científicas e tecnológicas do Ensino Médio e da Educação Profissional (BRASIL, 2000), o que foi reafirmado em 2004 pelo Decreto ${ }^{\circ} 5.224$ (BRASIL, 2004). A partir disso, essas instituições passaram a implantar cursos de licenciatura, porém, segundo Bonfim (2003), em condições diferentes das Universidades, tradicionalmente engajadas nessa missão. Por isso, inicialmente a formação de professores nos CEFET foi marcada por uma racionalidade hegemônica de se fazer mais com menos, de implementação de um processo aligeirado sem quadro docente e acervo bibliográfico específico, por exemplo (BONFIM, 2003).

Ou seja, logo de início os IF já possuíam alguns cursos de licenciaturas e, consequentemente, alguma experiência adquirida. Entretanto, por força de sua Lei de Criação (BRASIL, 2008) - nomeadamente o Art. $8^{\circ}$ que trata da reserva de $50 \%$ de suas vagas para a educação profissional técnica e $20 \%$ de suas de vagas para as licenciaturas, os IF precisaram ao 

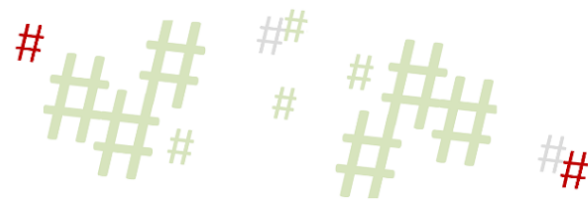

mesmo tempo ampliar significativamente o quantitativo de cursos de licenciaturas e manter o predomínio para a oferta de cursos técnicos profissionalizantes.

Passados dez anos da criação dos IF, em termos de números de cursos em funcionamento, registramos que, simultaneamente, os IF preservam sua característica histórica de ofertarem cursos voltados para a formação técnica e profissional e incorporou em sua matriz institucional as licenciaturas, nomeadamente nas áreas de Ciências e Matemática.

Pensando em uma razão que impôs aos IF uma normativa quantitativa para os cursos de formação de professores, temos uma recorrente relação com a escassez de professores no Ensino Médio (LIMA, 2016; ARAÚJO; MOURÃO, 2018). Além disso, há uma indicação para que as licenciaturas dos IF sejam voltadas para a áreas de Ciências e Matemática, que, por sua vez, eram áreas com elevado déficit de professores (BRASIL, 2009b; ARAUJO; VIANNA, 2011). Tal déficit ainda continua, segundo recentes censos escolares da educação básica (BRASIL, 2018d; BRASIL, 2020), mas com o importante registro do crescimento percentual de professores com formação superior em licenciatura ou bacharelado com complementação pedagógica atuantes na mesma área da disciplina que leciona.

Do ponto de vista quantitativo, podemos considerar as licenciaturas existentes nos IF como algo positivo, uma vez que nos últimos dez anos essas instituições têm formado professores em seus campis espalhados pelo país e, consequentemente, contribuído com o problema de escassez. Todavia, conforme Gatti, Barreto e André (2011) nos alertam, a escassez de professores é um problema histórico brasileiro, recorrentemente enfrentado por medidas emergenciais, tais como cursos rápidos de formação docente, complementação de formações de origens diversas (capacitações docentes), autorizações especiais para exercício do magistério a não licenciados, admissão de professores leigos, entre outras medidas. Em outras palavras, a escassez de professores devidamente preparados para atuarem na educação básica é uma questão estrutural, um problema crônico (FREITAS, 2007).

Em vista disso, não podemos pensar que o enfrentamento à escassez de professores na educação básica é de domínio restrito das instituições formadoras, já que isso seria negligenciar a totalidade desse problema. $\mathrm{O}$ aumento da oferta de cursos para a formação docente será insuficiente, se esta não vier acompanhada de outros fatores que interferem nos altos índices de evasão, tanto de estudantes matriculados em cursos de licenciaturas quanto de professores formados que não ingressam ou deixam precocemente a profissão (ARAUJO; VIANNA, 2011; PINTO, 2014). É nesse sentido que precisamos de políticas públicas direcionadas para uma reestruturação da formação e da carreira docente, que reconheçam o valor social dessa profissão (SHEIBE, 2010; KUENZER, 2011; ANDRÉ, 2015).

Por isso, retomando nosso olhar para as licenciaturas existentes nos IF, entendemos que a elevada oferta destes cursos é apenas um dos aspectos necessários a serem impulsionados, não podendo ser apenas justificada pela quantidade: é necessário garantir uma boa qualidade dessa formação. Nessa perspectiva, temos que possivelmente muitas das licenciaturas hoje em funcionamento nos IF surgiram a partir de uma exigência legal, Lei no 11.892/2008 (BRASIL, 2008), e que, assim como no processo de inserção de licenciaturas nos CEFET (BONFIM, 2003), foram implementadas em regime de urgência, em condições pouco favoráveis para o desenvolvimento pleno da formação docente.

Soma-se a essa conjuntura a complexidade de se adequar aos marcos regulatórios para os cursos de formação de professores, visto que estes passaram por importantes alterações (BRASIL, 2001; BRASIL, 2015) e ainda acenam para uma nova dinâmica devido à aprovação da Base Nacional Comum Curricular (BNCC) (BRASIL, 2017). Ou seja, no horizonte dos IF, o grande desafio que se apresenta para as licenciaturas é de consolidação, de se afirmarem como 
cursos de excelência no campo da formação docente. Para tal, é preciso percorrer um caminho de sintonia entre as demandas institucionais, particularidades do campus, e as normativas legais, documentos que regulam as licenciaturas (SECCO; REBEQUE; SOUZA, 2017).

Naturalmente, a exigência normativa da Lei nº 11.892/2008 (BRASIL, 2008) é um fator importante na tomada de decisão para a abertura de um curso de licenciatura nos IF. Segundo o levantamento de caráter quantitativo que realizamos neste artigo, sobre os cursos em funcionamento nos IF, vale destacarmos o engajamento dos IF na criação de licenciaturas, o que nos leva a crer uma afirmação dessas instituições no campo da formação de professores, ofertando cursos, em sua maioria, na modalidade presencial e na região Nordeste do Brasil.

Dessa forma, é preciso considerar que a oferta pública e gratuita de licenciaturas nos IF é uma conquista, pois busca assegurar oportunidades de formação docente em nível superior nas regiões economicamente menos dinâmicas. Portanto, a configuração de oferta que os IF representam, ao expandir seus cursos de formação de professores, é de uma iniciativa em prol da educação pública, realizada pelos diversos campi dos IF que muitas vezes se apresentam como única alternativa de formação superior pública de uma cidade ou de uma microrregião.

Para continuarmos nossas pesquisas sobre as licenciaturas nos IF precisamos ir além do simples olhar quantitativo de cursos em funcionamento, da averiguação do cumprimento da exigência legal de ofertar cursos de licenciaturas. Nesse sentido, entendemos que uma das alternativas para melhor entendimento do papel desempenhado pelas licenciaturas nos IF está no desenvolvimento de investigações sobre específicos cursos (tais como os estudos de caso). Quer dizer, para avançarmos no entendimento sobre a consolidação de um específico curso de licenciatura do IF precisamos considerar, por exemplo, indicadores de ingresso, de evasão e de conclusão, questões relacionadas ao campus (infraestrutura, composição do corpo docente, matriz orçamentária, entre outros) e ao projeto pedagógico do curso, assim como os impactos na vida dos egressos, se estes foram atuar na educação básica, ou cursar pós-graduação, ou ainda, abandonaram a carreira docência.

\section{Financiamento e agradecimentos}

O primeiro autor agradece ao campus Bento Gonçalves do Instituto Federal de Educação, Ciência e Tecnologia do Rio Grande do Sul pelo apoio financeiro da bolsa de estudos concedida através do Edital complementar IFRS/BG - $\mathrm{N}^{\circ}$ 51/2018 vinculado ao Edital IFRS/PROPPI - N 77/2018 - Fomento Interno 2019/2020.

\section{Referências}

ANDRÉ, M. Políticas de valorização do trabalho docente no Brasil: algumas questões. Ensaio: avaliação e políticas públicas em educação, Rio de Janeiro, v. 23, n. 86, p. 213-230, 2015.

ARAUJO, R. S.; VIANNA, D. M. A carência de professores de Ciências e Matemática na educação básica e a ampliação das vagas no ensino superior. Ciência \& Educação, Bauru, v. 17, n. 4, p. 807-822, 2011.

ARAÚJO, J. J. C. N.; MOURÃO, A. R. B. Estado de conhecimento sobre a formação de professores para a Educação Básica em Institutos Federais. In: SHIGUNOV NETO, A.; 
SILVA, A. C.; FORTUNATO, I. (org.). Passado, Presente e Futuro nos Institutos Federais de Educação. São Paulo: Edições Hipóteses, 2018.

BONFIM, M. I. (coord.). A formação docente nos centros federais de educação tecnológica: diagnóstico sobre a oferta das licenciaturas nos CEFETs. Brasília: MEC/SETEC, 2003.

BRASIL. Decreto $n^{\circ}$ 3.462, de 17 de Maio 2000: Dá nova redação ao art. 8o do Decreto no 2.406, de 27 de novembro de 1997, que regulamenta a Lei $\mathrm{n}^{\circ}$ 8.948, de 8 de dezembro de 1994. Brasília, 2000. Disponível em: http://www.planalto.gov.br/ccivil_03/decreto/ d3462.htm. Acesso em 11 de fevereiro de 2020.

BRASIL. Parecer CNE/CP N 9/2001: Diretrizes curriculares nacionais para a formação de professores da educação básica, em nível superior, curso de Licenciatura, de graduação plena. Brasília, 2001. Disponível em: http://portal.mec.gov.br/cne/arquivos/pdf/009.pdf. Acesso em 12 de fevereiro de 2020.

BRASIL. Decreto ${ }^{\circ}$ 5.224, de 1 de Outubro de 2004: Dispõe sobre a organização dos Centros Federais de Educação Tecnológica e dá outras providências. Brasília, 2004. Disponível em: http://www.planalto.gov.br/ccivil_03/_Ato2004-2006/2004/Decreto/ D5224.htm\#art31. Acesso em 11 de fevereiro de 2020.

BRASIL. Lei no 11.892, de 29 de Dezembro de 2008: Institui a Rede Federal de Educação Profissional, Científica e Tecnológica, cria os Institutos Federais de Educação, Ciência e Tecnologia, e dá outras providências. Brasília, 2008. Disponível em: http://www.planalto. gov.br/ccivil_03/_Ato2007-2010/2008/Lei/L11892.htm. Acesso em 14 de agosto de 2019.

BRASIL. Centenário da Rede Federal de Educação Profissional e Tecnológica. Brasília, 2009a. Disponível em: http://portal.mec.gov.br/setec/arquivos/centenario/historico_educacao_ profissional.pdf. Acesso em 08 de julho de 2019.

BRASIL. Estudo exploratório sobre o professor brasileiro com base no Censo Escolar da Educação Básica 2007. Instituto Nacional de Estudos e Pesquisas Educacionais Anísio Teixeira (INEP). Brasília, 2009b. Disponível em: http://portal.mec.gov.br/dmdocuments/ estudoprofessor.pdf. Acesso em 12 de fevereiro de 2020.

BRASIL. Resolução CNE $\mathbf{N}^{\circ}$ 2/2015: Define as Diretrizes Curriculares Nacionais para a formação inicial em nível superior (cursos de licenciatura, cursos de formação pedagógica para graduados e cursos de segunda licenciatura). Brasília, 2015. Disponível em: http://portal.mec.gov.br/docman/agosto-2017-pdf/70431-res-cne-cp-002-03072015-pdf/file. Acesso em 12 de fevereiro de 2020.

BRASIL. Resolução CNE/CP N²/2017: Instituiu e orienta a implantação da Base Nacional Comum Curricular, a ser respeitada obrigatoriamente ao longo das etapas e respectivas modalidades no âmbito da Educação Básica. Brasília, 2017. Disponível em: http://basenacionalcomum.mec.gov.br/images/historico/RESOLUCAOCNE_CP222DEDEZE MBRODE2017.pdf. Acesso em 12 de fevereiro de 2020. 
BRASIL. Portaria ${ }^{\circ}$ 1, de 3 de Janeiro de 2018: Institui a Plataforma Nilo Peçanha - PNP, a Rede de Coleta, Validação e Disseminação das Estatísticas da Rede Federal de Educação Profissional, Científica e Tecnológica. Brasília, 2018a. Disponível em: http://portal.mec.gov. br/component/content/article?id=67961. Acesso em 4 de março de 2020.

BRASIL. Portaria ${ }^{\circ}$ 1, de 21 de Novembro de 2018: Define conceitos e estabelece fatores para uso na Plataforma Nilo Peçanha para cálculo dos indicadores de gestão das Instituições da Rede Federal de Educação Profissional, Científica e Tecnológica. Brasília, 2018b. Disponível: http://portal.mec.gov.br/component/content/article?id=67961. Acesso em 4 de março de 2020.

BRASIL. Portaria n ${ }^{\circ} 1.162$, de 9 de Novembro de 2018: Regulamenta o conceito de AlunoEquivalente e de Relação Aluno por Professor, no âmbito da Rede Federal de Educação Profissional, Científica e Tecnológica. Brasília, 2018c. Disponível em: http://portal.mec.gov. br/component/content/article?id=67961. Acesso em 4 de março de 2020.

BRASIL. Censo Escolar 2017: notas estatísticas. Instituto Nacional de Estudos e Pesquisas Educacionais Anísio Teixeira (INEP). Brasília, 2018d. Disponível em: http://download.inep. gov.br/educacao_basica/censo_escolar/notas_estatisticas/2018/notas_estatisticas_Censo_Esco lar_2017.pdf. Acesso em 12 de fevereiro de 2020.

BRASIL. Censo Escolar 2019: notas estatísticas. Instituto Nacional de Estudos e Pesquisas Educacionais Anísio Teixeira (INEP). Brasília, 2020. Disponível em: http://portal.inep.gov.br/ documents/186968/0/Notas+Estat\%C3\%ADsticas+-+Censo+da+Educa\%C3\%A7\%C3\%A3o+ B\%C3\%A1sica+2019/43bf4c5b-b478-4c5d-ae17-7d55ced4c37d?version=1.0. Acesso em 12 de fevereiro de 2020.

CASTIONI, R.; SOUZA, E. C. L. (org). Institutos Federais: os desafios da institucionalização. Brasília: Editora Universidade de Brasília, 2012.

DINIZ-PEREIRA, J. E. A situação atual dos cursos de licenciatura no Brasil frente À hegemonia da educação mercantil e empresarial. Revista Eletrônica de Educação, São Carlos, v. 9, n. 3, p. 273-280, 2015.

FREITAS, Helena Costa Lopes de. A (nova) política de formação de professores a prioridade postergada. Educação \& Sociedade. Campinas, v. 28, n. 100 - esp., p. 1203-1230, 2007.

FRIGOTTO, G. Indeterminação de Identidade e reflexos nas políticas institucionais formativas dos IFs. In: FRIGOTTO, G. (org.). Institutos Federais de Educação, Ciência e Tecnologia: relação com o ensino médio integrado e o projeto societário de desenvolvimento. Rio de Janeiro: Editora UERJ, 2018.

GATTI, B. A.; BARRETO, E. S.; ANDRÉ, M. E. D. Políticas Docentes no Brasil: um estudo da arte. Brasília: UNESCO, 2011.

JURACY, C. (org.). Institutos Federais Lei 11.892, de 29/12/2008: comentários e reflexões. Natal: Editora IFRN, 2009. 
KUENZER, A. Z. A formação de professores para o ensino médio: velhos problemas, novos desafios. Educação \& Sociedade, Campinas, v. 32, n. 116, p. 667-688, 2011.

LIMA, F. B. G. Os Institutos Federais como um novo locus de formação de professores. Movimento - revista de educação, Niterói, v. 3, n. 4, p. 297-325, 2016.

LIMA, F. B. G.; CURADO SILVA, K. A. C. P. C. A concepção de formação de professores nos Institutos Federais: um estudo dos documentos oficiais. Revista da Faculdade de Educação, Cáceres, v. 20, ano 11, n. 2, p. 15-33, 2013.

MORAES, G. H. et al. Plataforma Nilo Peçanha: guia de referência metodológica. Brasília: Ed. Evobiz, 2018. Disponível em: http://portal.mec. gov.br/plataforma-nilopecanha/plataforma-nilo-pecanha-guia-de-eferencia-metodologica. Acesso em 4 de março de 2020 .

PINTO, J. M. R. O que explica a falta de professores nas escolas brasileiras? Jornal de Políticas Educacionais, Curitiba, N 15, p. 03-12, 2014.

SECCO, D.; REBEQUE, P. V.; SOUZA, J. Análise da evolução dos projetos pedagógicos de um curso de formação inicial de professores de Física. \#Tear: Revista de Educação, Ciência e Tecnologia, Canoas, v. 6, n. 2, p. 1-21, 2017.

SHEIBE, L. Valorização e formação dos professores para a educação básica: questões desafiadoras para um novo plano nacional de educação. Educação \& Sociedade, Campinas, v. 31, n. 112, p. 981-1000, 2010.

ShigunOV NeTO, A.; SILVA, A. C.; FORTUNATO, I. (org.). Passado, Presente e Futuro nos Institutos Federais de Educação. São Paulo: Edições Hipóteses, 2018.

Recebido em março de 2020.

Aprovado em maio de 2020. 